\title{
Feasibility of Cellular Networks and Radar Coexistence in S Spectrum Band
}

\author{
Sabogu-S Raymond, \\ Hanbat National University \\ Department of \\ Electronic Eng. \\ Daejeon, South Korea
}

\author{
Derek Pobi Asiedu \\ Hanbat National University \\ Department of \\ Electronic Eng. \\ Daejeon, South Korea
}

\author{
Sumaila Mahama \\ Hanbat National University \\ Department of \\ Electronic Eng. \\ Daejeon, South Korea
}

\author{
Han-Shin Jo \\ Hanbat National University \\ Department of \\ Electronic Eng. \\ Daejeon, South Korea
}

\begin{abstract}
Spectrum sharing is an efficient solution for spectrum shortage. This paper presents mathematical analysis of a typical coexistence scenario between LTE and radar systems in 2.7-2.9 $\mathrm{GHz}$ bands. We present the interference analysis of multiple base stations (BSs) and mobile stations (MSs) on the primary radar. The joint distribution of power-controlled and non powercontrolled interference is also presented in this study. Our simulation and analytical results closely approximate each other and the LTE downlink data rate for the shared spectrum is quite comparable to an LTE system operating in a dedicated spectrum band. Thus, the feasibility of spectral coexistence is shown.
\end{abstract}

\section{General Terms}

Spectrum Sharing, Coexistence

\section{Keywords}

Spectrum sharing, power control, Poisson, Log-normal distribution.

\section{INTRODUCTION}

The advent of $5 \mathrm{G}$ requires sufficient spectrum to make it a reality. The current form of spectrum allocation is static which has led to the inefficient utilization of this precious resource (ie spectrum). Federal communication commission (FCC) and other world telecommunication regulatory bodies have identified huge chunks of spectrum held by fixed wireless communication services been underutilized. Spectrum sharing is a promising technique that will help in harnessing more spectrums for the 5G systems. Much study has been done on this important subject. Raymond et al in [1] studied spectral coexistence between radar systems and powercontrolled cellular systems in the $2.7-2.9 \mathrm{GHz}$ bands. They devised mechanisms to ensure secondary access to this band by cellular systems. M.I Rahman in [2] also studied coexistence between LTE-BS and radar systems considering only the radar main beam gain. An account was not made for the radar antenna sidelobes and its time varying gain due to the rotation. M. Tercero et al in [3] analyzed the impact of aggregate interference on the primary radar mathematical using log-normal approximations without incorporating a guard region for the secondary users (SUs). In [4], a qualitative analysis was done indicating the possibility of sharing radar spectrum with other wireless services. Other researchers in [5] proposed the projection of the signals of the two systems in the null space of each using a blind learning approach. The authors of [5-10] presented other techniques in analyzing spectral coexistence between radar systems and other wireless communication services.

This paper presents mathematical modeling of the probability distribution of power control (PC) and non (PC) interference from cellular BSs or MSs using joint log normal and Poisson distributions. To the best of our knowledge this is the first paper that considers joint interference distribution as the authors of [1] and [3] separately model the two scenarios. The rest of the paper is organized as follows; we present mathematical derivation of the joint probability distribution functions of the PC and non PC BSs and MSs interference on the primary radar system in section 2 . In section 3 , we present the results showing the feasibility of spectral coexistence in the $\mathrm{S}$ spectrum band with cellular systems and section 4 concludes the paper..

\section{PROBABILITY DISTRIBUTION OF INTERFERENCE}

\subsection{Joint Probability Distribution of BSs Interference on the Radar}

The authors of [1] analyzed the distribution of the interference from power controlled BSs on the primary radar. This section of the paper models the joint distribution of power controlled BSs and non power controlled BSs interference mathematically. The representation of this model is depicted in Fig.1. BSs in the annular region $\mathrm{R} 2-\mathrm{R} 1$ activates the power control algorithm developed in [1] to prevent harmful interference on the radar system whereas those in R3-R2 operates normally as an LTE system operating in a dedicated spectrum band. The probability distribution functions of the separation distances of PC and no PC BSs from the radar $\left(d_{k 1}, d_{k 2}\right)$ are given by (1) and (2) respectively as

$$
\begin{aligned}
& f_{d_{k 1}}\left(x_{1}\right)=\frac{2 x_{1}}{R_{2}^{2}-R_{1}^{2}}, R_{1} \leq x_{1} \leq R_{2}, \\
& f_{d_{k 2}}\left(x_{2}\right)=\frac{2 x_{2}}{R_{3}^{2}-R_{1}^{2}}, R_{2} \leq x_{2} \leq R_{2} .
\end{aligned}
$$




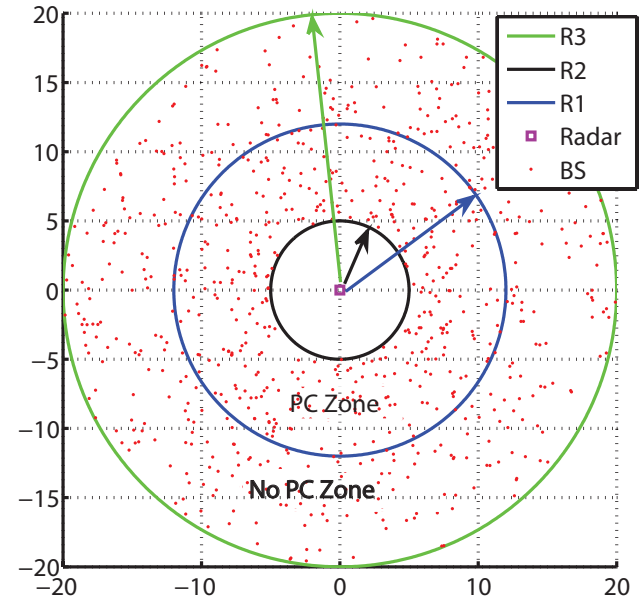

Fig. 1. Representation of Multiple BSs Interference scenario.

The PDF of the arbitrary interference $\gamma_{k 1}$ and $\gamma_{k 2}$ from PC and no PC BSs are respectively derived as

$$
f_{\gamma_{k 1}}(z)=\int_{z / Q_{1}}^{z / Q_{2}} \frac{1}{\left|x_{1}\right|} f_{x_{1 k 1}}\left(x_{1}\right) f_{U_{1 k 1}}\left(\frac{z}{x_{1}}\right) d x_{1}
$$

and

$$
f_{\gamma_{k 2}}(z)=\int_{z / Q_{2}}^{z / Q_{3}} \frac{1}{\left|x_{2}\right|} f_{x_{2 k 2}}\left(x_{2}\right) f_{U_{2 k 2}}\left(\frac{z}{x_{2}}\right) d x_{2},
$$

where $\gamma_{k 1}$ and $U_{k 1}$ are defined respectively as $\gamma_{k 1}=U_{1 k X_{k}}=$ $P_{T} G L\left(d_{k 1}\right) X_{k 1}, R_{1} \leq d_{k 1} \leq R_{2} \cdot \gamma_{k 2}$ and $U_{k 2}$ are similarly defined with only a change in the subscript from 1 to 2 . $G=$ $G_{t} G_{r} / 10^{\mathrm{FDR} / 10}$ and $L\left(d_{k}\right)=L_{o}\left(\frac{d_{k}}{d_{o}}\right)^{-n}, d_{o} \leq R_{1} \leq R_{2} . P_{T}$, $G_{t}$ and $G_{r}$ are the BS transmit power, maximum antenna gains of the BS and radar respectively, whereas $L_{o}, d_{o}$ and $n$ are the path loss, reference distance and path loss exponent respectively as similarly given in [1].

The joint PDF of the PC and no PC interference is derived from (3) and (4) as

$$
f_{\gamma_{k 1}, \gamma_{k 1}}(z)=\int_{z / Q_{2}}^{z / Q_{3}} \int_{z / Q_{2}}^{z / Q_{3}} f_{\gamma_{k 1}, \gamma_{k 2}}\left(x_{1}, x_{2}\right) d x_{1} d x_{2} .
$$

The solutions of (3-5) are respectively given by (6), (9) and (13) as similarly derived in [11] .

$$
S_{1}=\Omega_{z^{\frac{-2}{n}-1}}\left(A_{1}-B_{1}\right),
$$

where

$$
\begin{aligned}
& A_{1}=\operatorname{erf}\left(\frac{\ln \left(z / Q_{2}\right)-2 \sigma_{x_{1 k 1}}^{2} / n}{\sqrt{2 \sigma_{x_{1} k_{1}}^{2}}}\right), \\
& B_{1}=\operatorname{erf}\left(\frac{\ln \left(z / Q_{1}\right)-2 \sigma_{x_{1} k_{1}}^{2} / n}{\sqrt{2 \sigma_{x_{1} k_{1}}^{2}}}\right),
\end{aligned}
$$

$\Omega_{1}=\frac{1}{R_{2}^{2}-R_{1}^{2}}\left(\frac{d_{o}^{-n}}{G P_{t} L_{o}}\right)^{\frac{-2}{n}} \exp \left(2 \sigma_{x_{1} k_{1}}^{2} / n^{2}\right) \quad, \quad Q_{1}=$ $G P_{t} L_{o}\left(R_{1} / d_{o}\right)^{-n}$ and $Q_{2}=G P_{t} L_{o}\left(R_{2} / d_{o}\right)^{-n}$.

$$
S_{2}=\Omega_{z}^{\frac{-2}{n}-1}\left(A_{2}-B_{2}\right)
$$

where

$$
\begin{gathered}
A_{2}=\operatorname{erf}\left(\frac{\ln \left(z / Q_{3}\right)-2 \sigma_{x_{k}}^{2} / n}{\sqrt{2 \sigma_{x_{2} k_{2}}^{2}}}\right), \\
B_{2}=\operatorname{erf}\left(\frac{\ln \left(z / Q_{2}\right)-2 \sigma_{x_{2} k_{2}}^{2} / n}{\sqrt{2 \sigma_{x_{2} k_{2}}^{2}}}\right), \\
\Omega_{2}=\frac{1}{R_{3}^{2}-R_{2}^{2}}\left(\frac{d_{o}^{-n}}{G P_{t} L_{o}}\right)^{\frac{-2}{n}} \exp \left(2 \sigma_{x_{2} k_{2}}^{2} / n^{2}\right) \quad, \quad Q_{2} \\
\text { and } Q_{3}=G P_{t} L_{o}\left(R_{3} / d_{o}\right)^{-n} . \\
R_{\gamma_{k_{1}}, \gamma_{k_{1}}}(z)=\int_{\left.z / d_{o}\right)^{-n}}^{z / Q_{3}} \int_{z / Q_{1}}^{z / Q_{2}} \frac{1}{\left|x_{1}\right|} f_{x_{k_{1}}}\left(x_{1}\right) f_{U_{k_{1}}} \\
\left(\frac{z}{x_{1}}\right) \frac{1}{\left|x_{2}\right|} f_{x_{k_{2}}}\left(x_{2}\right) f_{U_{k_{2}}}\left(\frac{z}{x_{2}}\right) d x_{1} d x_{2} .
\end{gathered}
$$

Since $S_{1}$ is a constant with respect to $S_{2}$ the solution of above integral becomes

$$
\begin{aligned}
S_{3}= & \int_{z / Q_{2}}^{z / Q_{3}} S_{1} \frac{1}{\left|x_{2}\right|} f_{x_{k_{2}}}\left(x_{2}\right) f_{U_{k_{2}}}\left(\frac{z}{x_{2}}\right) d x_{2} \\
& S_{1} \int_{z / Q_{2}}^{z / Q_{3}} \frac{1}{\left|x_{2}\right|} f_{x_{k_{2}}}\left(x_{2}\right) f_{U_{k_{2}}}\left(\frac{z}{x_{2}}\right) d x_{2} \\
& =S_{1} S_{2}
\end{aligned}
$$

\subsection{Mathematical Analysis of MSs Interference on Primary Radar}

The arrival of MSs at their respective serving BSs follows a Poisson process with a mean arrival rate denoted by $\mu$ and the number of MSs arriving at the BSs is K. Without the loss of generality, the distribution of the interference from the multiple MSs follows the Poisson distribution. The probability distribution of the random MSs is modeled as

$$
P_{k}=\frac{e^{-\mu} \mu^{k}}{k !}
$$

Denoting $\zeta_{k}$ as the interference from an arbitrary arriving MS. The probability distribution of the interference is similarly given as

$$
P_{\zeta_{k}}=\frac{e^{-\mu_{\zeta_{k}}} \mu_{\zeta_{k}}^{k}}{k !}
$$


The mean and variance of the random variable $\zeta_{k}$ are computed from the first and second moments respectively in (8) and (10).

$$
\begin{aligned}
E\left[\zeta_{k}\right]= & \sum_{k=0}^{N} K P_{k}=\sum_{k=0}^{N} \frac{K e^{-\mu_{\zeta_{k}}} \mu_{\zeta_{k}}^{k}}{k !} \\
& =\mu_{\zeta_{k}} e^{-\mu_{\zeta_{k}}} \sum_{k=1}^{N} \frac{\mu_{\zeta_{k}}^{k-1}}{(k-1) !}, \text { but } \sum_{k=1}^{N} \frac{\mu_{\zeta_{k}}^{k-1}}{(k-1) !}=e^{\mu_{\zeta_{k}}} \\
& =\mu_{\zeta_{k}} e^{-\mu_{\zeta_{k}}} e^{\mu_{\zeta_{k}}}=\mu_{\zeta_{k}},
\end{aligned}
$$

where $\mathrm{N}$ is the sum of all interfering MSs. The variance of the interference is given as the second moment. We first evaluate

$$
\begin{aligned}
E\left[\zeta_{k}\left(\zeta_{k}-1\right)\right] & =\sum_{k=2}^{N} K(K-1) P_{k} \\
& \mu_{\zeta_{k}}^{2} e^{-\mu_{\zeta_{k}}} \sum_{k=2}^{N} \frac{\mu_{\zeta_{k}}^{(k-2)}}{(k-2) !} \\
& =\mu_{\zeta_{k}}^{2}, \text { because } \sum_{k=2}^{N} \frac{\mu_{\zeta_{k}}^{(k-2)}}{(k-2) !}=e^{\mu_{\zeta_{k}}}
\end{aligned}
$$

The variance of the interference then computed as

$$
\begin{aligned}
& \sigma_{\zeta_{k}}^{2}=\operatorname{Var}\left[\zeta_{k}^{2}\right]-E\left[\zeta_{k}\right]^{2}, \\
& \quad \text { but, } E\left[\zeta_{k}^{2}\right]=E\left[\zeta_{k}\left(\zeta_{k}-1\right)+E\left[\zeta_{k}\right]=\mu_{\zeta_{k}}^{2}+\mu_{\zeta_{k}}\right. \\
& \quad \text { therefore },=\mu_{\zeta_{k}}^{2}+\mu_{\zeta_{k}}-\mu_{\zeta_{k}}^{2}=\mu_{\zeta_{k}}
\end{aligned}
$$

Thus, the mean and variance of the MSs interference on the radar are equal. The aggregate interference $\left(I_{a g g}\right)$ on the PU from all MSs is given as

$$
I_{\text {agg }}=\sum_{k=1}^{N} \zeta_{k}
$$

As the mean number of the arriving MSs increases, the distribution of their interference on the radar system gets more symmetrical. In light of the above-mentioned and without any ambiguity, we assumed the interference is log-normally distributed based on the central limit theorem for independent and identically distributed random variables. The Log-normal CDF of the interference is approximated using (25) in [1].

\section{NUMERICAL RESULTS}

The results of [1] gives the required separation distances coexistence between the primary radar and the secondary LTE system. We assumed a perfectly planned cellular system with dominant interference from the radar system located at a maximum range of $100 \mathrm{~km}$ and a rayleigh fading channel between the LTE BS and MS. The interference from the radar system is computed using (2) in [1] and applying the parameters of tables I and II in [1]. Exclusion radii of $\mathrm{R} 1=4 \mathrm{~km}$ and $\mathrm{R} 2=6 \mathrm{~km}$ are used for urban and $\mathrm{R} 1=10$ $\mathrm{km}$ and $15 \mathrm{~km}$ for suburban (based on the results of [1]). The ITUR P.1456 model is used to capture the propagation losses between the radar and the LTE system.

The cellular system's cell edge downlink data rate for MSs located in the power control zone is lower than that of the non power control users as depicted in Fig.2. This is expected because of the low transmit power at the BS during the power control period. It is also observed that, the performance in the urban environment is better

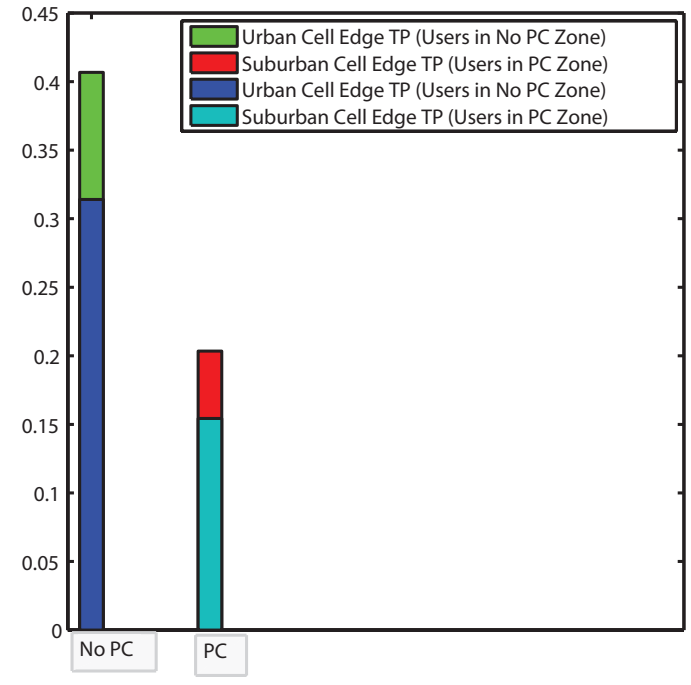

Fig. 2. SU Cell Edge throughput

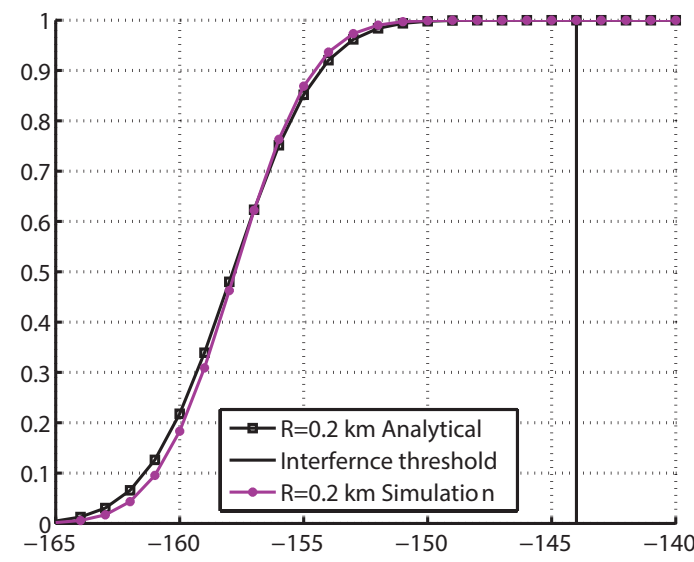

Fig. 3. Multiple MSs Interference Scenario

compared to the suburban which is desirable because of the spectrum demand for high broadband applications is in the urban. An interference-to-noise ratio of - $10 \mathrm{~dB}$ (correspond to an interference threshold $\left(I_{t h r}\right)$ of $\left.-144 \mathrm{dBW}\right)$ is used for optimum protection of the radar system from interference emanating from multiple MSs. Our simulation results closely follow the analytical results obtained from (25) in [1] indicating a radar system protection radius requirement of 0.2 for multiple MSs coexistence.

\section{CONCLUSION}

This paper considers secondary spectrum access to radar bands by cellular systems. We analyze the joint probability distribution of power control and non power control SUs interference on the PU mathematically. Typical parameters of ASR-11 are used to proof the feasibility of our analysis. The performance of the SU systems in terms of cell edge throughput is comparable to an SU operating 
in a dedicated spectrum and hence we conclude that coexistence in the $\mathrm{S}$ band spectrum is feasible. The authors intend extending this work into millimeter wave spectrum band in the future.

\section{ACKNOWLEDGMENT}

This research was supported by the research fund of Hanbat National University in 2016

\section{REFERENCES}

[1] S-S. Raymond et al., "Coexistence of power-controlled cellular networks with rotating radar,'IEEE J. Sel. Areas Commun., vol. PP., iss. 99, 2016.

[2] M.I Rahman, J.S. Karlsson, "Feasibility evaluations for secondary LTE usage in $2.7-2.9 \mathrm{GHz}$ radar bands," IEEE International symposium on personal, Indoor and Mobile Radio Communications, 2011.

[3] M. Tercero, K.W Sung and J.Z, 'Impact of aggregate Interference on Meteorological Radar from Secondary users," IEEE Wireless Communications and Networking Conference, March 2011.

[4] E. Obregon, K.W Sung, and J.Zander, "On the Sharing Opportunities for Ultra-Dense Networks in the Radar Bands,'IEEE International Symposium on Dynamic Spectrum Access Networks (DYSPAN), 2014.

[5] S-S. Raymond, A. Abubakari, H-S. Jo, H-J. Hong and H.Kyung Son, "Compatibility between LTE and Airport Surveillance Radar in 2700-2900 MHz radar bands," Int. Conference on ICT Convergence, (ICTC), 2015.

[6] R. Saruthirathanaworakun, J. M. Peha, and L. M. Correia, "Opportunistic Sharing Between Rotating Radar and Cellular," IEEE Journal on Selected Areas in Communications, Vol. 30, NO. 10, November 2012.

[7] Joel T. Johnson, Christopher J. Baker, Huaiyi Wang, Lixin Ye, and Chenglin Zhang, "Assessing the Potential for Spectrum Sharing between Communications and Radar Systems in the L-Band Portion of the RF Spectrum Allocated to Radar," International Conference on Electromagnetics in Advanced Applications (ICEAA), August 2014.

[8] Rathapon Saruthirathanaworakun, Jon M. Peha, and Luis M. Correia, "Gray-Space Spectrum Sharing between Multiple Rotating Radars and Cellular Network Hotspots," IEEE Vehicular Technology Conference (VTC) Spring, 2013.

[9] Awais Khawar, Ahmed Abdel-Hadi, and T. Charles Clancy, "Spectrum Sharing between S-band Radar and LTE Cellular System: A Spatial Approach," IEEE International Symposium on Dynamic Spectrum Access Networks: SSPARC Workshop, April 2014.

[10] Michael J. Marcus, "Sharing Government Spectrum with Private Users: Opportunities and Challenges", IEEE Wireless Communications, June 2009. 